\title{
AN IMPROVED METHOD OF AGM FOR HIGH PRECISION GEOLOCATION OF SAR IMAGES
}

\author{
Guoqing Zhou ${ }^{1}$, Chaoshuang $\mathrm{He}^{1}$, Tao Yue ${ }^{1, *}$, Wei Huang ${ }^{1,2}$, Yu Huang ${ }^{1}$, Xiaozhu Li $^{1}$, Yutian Chen ${ }^{1}$ \\ ${ }^{1}$ Guangxi Key Laboratory of Spatial Information and Geomatics, Guilin University of Technology, No. 12 Jian'gan Road, Guilin, \\ Guangxi, 541004, China, (gzhou, yuetao)@glut.edu.cn \\ ${ }^{2}$ Department of Mechanical and Control Engineering, Guilin University of Technology, No. 12 Jian'gan Road, Guilin, Guangxi \\ 541004, China
}

Commission III, WG III/2

KEY WORDS: SAR image, Range-doppler, geolocation, AGM, linear regression

\begin{abstract}
In order to take full advantage of SAR images, it is necessary to obtain the high precision location of the image. During the geometric correction process of images, to ensure the accuracy of image geometric correction and extract the effective mapping information from the images, precise image geolocation is important. This paper presents an improved analytical geolocation method (IAGM) that determine the high precision geolocation of each pixel in a digital SAR image. This method is based on analytical geolocation method (AGM) proposed by X.K Yuan aiming at realizing the solution of RD model. Tests will be conducted using RADARSAT-2 SAR image. Comparing the predicted feature geolocation with the position as determined by high precision orthophoto, results indicate an accuracy of $50 \mathrm{~m}$ is attainable with this method. Error sources will be analyzed and some recommendations about improving image location accuracy in future spaceborne SAR's will be given.
\end{abstract}

\section{INTRODUCTION}

Synthetic Aperture Radar (SAR) is a kind of active microwave remote sensing technology, which can penetrate the earth surface and obtain measurement data all-day and all-weather. So it is appreciated by most countries in the world (G. Zhou, 2005). To make full use of the SAR image, it is necessary to obtain the high precision location of the image. The location information of the objects on SAR images is the basic information of the application of space-to-Earth observation technology such as topographic mapping, disaster monitoring, and resource census and change detection (J.P. Zhou, 2000). How to precisely achieve geolocation of the image has been the focus of photogrammetry and remote sensing. In addition, during the geometric correction process of images, in order to ensure the accuracy of image geometric correction and extract the effective mapping information from the images, precise image geolocation is also necessary.

The development of the geolocation of remote sensing is relatively tortuous. The study of remote sensing geolocation technology started with the theory of photogrammetry (J.P. Zhou, 2001, G. Zhou, 2016). It has gone through several stages at present and several common practices have been developed, which can be divided into three parts: (1) Establish the polynomial relation expression between the image coordinates and the geographic coordinates of corresponding actual objects, and use the large number of GCPs uniformly distributed in the image area to solve the polynomial coefficients so as to realize the pixel target geolocation (A. Loew, 2007). The advantage of this method is that the process is very simple. The computational efficiency is satisfied and the disadvantage is that a large number of control points are needed and the geolocation result in a region with a large undulation is poor and can not meet the requirements of high precision geolocation. It is entirely a mathematical approach without considering the SAR imaging mechanism. (2) At the 16th
ISPRS, well-known scholar Konecny (K. G., 1988) and others put forward the radar collinear equation model, which takes into account the changes of external orientation element of the sensor and the variation of the terrain fluctuation. Compared with the polynomial model, the imaging geometry is more rigorous and the geolocation result is more accurate. However, the model ignores the characteristics of the radar slant range projection, just simulating the radar imaging from the characteristics of the traditional optical imaging (G. Zhang, 2013, G. Zhou, 2017). (3) RD model developed by Curlander on the basis of Brown is based entirely on the imaging principle of SAR. This model consists of slant range equation, the Doppler frequency equation and the earth ellipsoid equation. Using this method, we input the image coordinate of a pixel, and obtain the a corresponding result of geodetic coordinates. The geolocation can be achieved without ground control point, the absolute geolocation accuracy is $200 \mathrm{~m}$ (J.C. CURLANDER, 1982).

In this paper, an improved analytical geolocation method (IAGM) has been presented to determine the high precision geolocation of each pixel in a digital SAR image. This method based on analytical geolocation method (AGM) proposed by X.K Yuan aims at realizing the solution of RD model (X.K. Yuan, 1998). However, the AGM is under the condition of assuming that a partial area just below the sub-satellite point is spherical. In fact the earth is not, which will lead to geolocation errors. The IAGM adds a condition of linear regression applied to correct geolocation. Linear regression describes the interdependence between two or more variables. In this paper, the linear regression describes a relationship between the difference and the AGM geolocation results. The difference is the result between the AGM geolocation results and the corresponding actual GCPs. The procedure of IAGM is as follows: Firstly, each pixel of the SAR image is calculated by using AGM algorithm. Secondly, we calculate the difference of

\footnotetext{
*Corresponding author: Tao Yue, Email: yuetao@glut.edu.cn.
} 
each pixel between the results obtained by using the AGM algorithm and the actual ground points coodinate. Thirdly, the difference equals the linear regression with the argument of the AGM geolocation results. Finally, we use sparse GCPs to calculate linear regression coefficients. Then the difference can be considered as the correction of the AGM geolocation results. The high precision geolocation of SAR images is realized by adding the coordinate correction value to the geolocation result of the AGM. To more clearly express IAGM principle, refer to the flowchart shown in Figure 1.

The second part mainly introduces the IAGM algorithm of the SAR image geolocation, including the introduction and analysis of the AGM. Then, based on the previous studies, the method is proposesed and discussed in detail. The third part conducts a experimental demonstration and dicussion based on the second part, including the experiment introduction, experimental process and result, the accuracy evaluation of experimental results and discussion. The fourth part draws conclusions and analyzes.

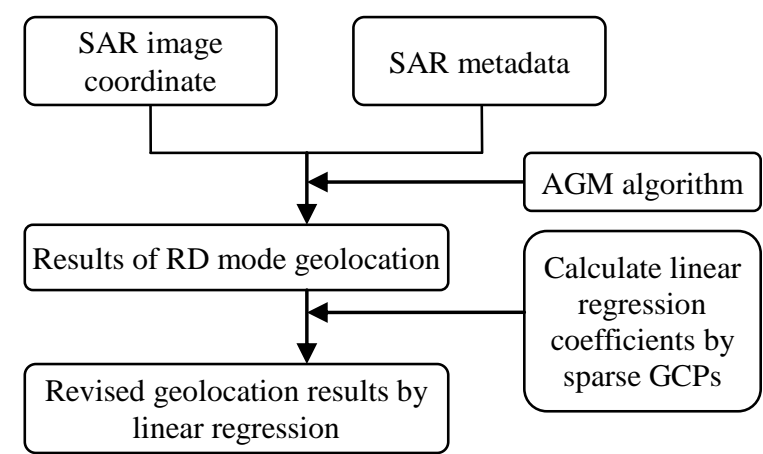

Figure 1. IAGM principle flowchart

\section{IAGM GEOLOCATION THEORY}

\subsection{RD Geolocation Model}

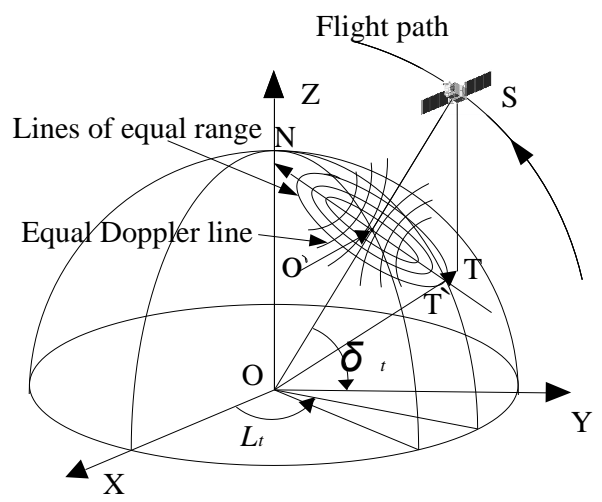

Figure 2. Geocentric Earth Fixed System (ECR)

2.1.1. Coordinate System for SAR Image Geolocation: Geocentric Earth Fixed System (ECR) will be used for the RD model geolocation. The definition is as follows: the center of Earth quality is regarded as the origin of coordinates, and the $\mathrm{X}$-axis points to the intersection between the equatorial plane and the Meridian surface that the Greenwich Observatory stays in. Z-axis points to north pole of Earth's ellipsoid, and the Y-axis is fixed by a right-handed Cartesian coordinate system. As shown in Figure 2, $\mathrm{S}$ is the position of the satellite, and $\mathrm{T}$ is the position of the object point. $\mathrm{T}^{\prime}$ is the projection point of $\mathrm{T}$ on the ellipsoid, and $\mathrm{O}$ is sub-satellite point. $\mathrm{L}_{\mathrm{t}}, \delta_{\mathrm{t}}$ are the geocentric latitude and longitude of the object point.
Image coordinate $(i, j)$ : $i$ represents the azimuthal imaging line coordinates of the SAR image, pointing to the flight path of the satellite, which exists the relationship of (1) with the imaging time $\mathrm{t}$ (Y.H. Zhang, 2001). j represents the range direction imaging column coordinates, which exists the relationship of (2) with slant range , and $t_{0}$ is the UTC time in the first line of SAR imaging, and $t_{1}$ is the sampling interval in azimuthal direction. Above parameters can be obtained from the metadata, and R ( $i$, j) represents the slant range between the actual object of the pixel $(i, j)$ on the SAR image and the phase center of the SAR antenna. $r_{0}$ is the near slant range, and $r_{1}$ is the range sampling interval. $\mathrm{r}_{0}, \mathrm{r}_{1}$ can also be read from the metadata.

$$
\begin{gathered}
t=t_{0}+t_{l} \times i \\
R(i, j)=r_{0}+r_{1} \times j
\end{gathered}
$$

Image pixel coordinate system $(\mathrm{m}, \mathrm{n})$ : $\mathrm{m}$ and $\mathrm{n}$ denote the row and column the pixel on the digital image whose origin locates upper left corner of the digital image. Image pixel coordinate system and image coordinate system are different due to different modes of SAR image when working (H.H. Xu, 2005). When the satellite running in way of descending and in way of left look, the two coordinate systems are the same. when the satellite in way of acsending and right look or in way of ascending and left look, $\mathrm{i}=\mathrm{m}$, and $\mathrm{j}=$ maximal pixel column number $-n$. When in way of ascending and right look, $\mathrm{j}=\mathrm{n}$, and $\mathrm{i}=$ maximal pixel line number $-\mathrm{m}$.

2.1.2. Description of Satellite Orbital State:The satellite orbital state vector includes the position vector and velocity vector of the antenna phase center of the SAR sensor carried by the satellite. In this paper, the orbit of the satellite stays under the coordinate system of ECR. Rs $=\left(X_{S}, Y_{S}, Z_{S}\right)$ and $V_{s}=\left(X_{V}\right.$, $\left.Y_{V}, Z_{V}\right)$ represent the position vector and velocity vector. To one scene of SAR image, metadata provides a number of satellite orbital states in different time t. It takes only several seconds to sweep the whole scene, so that it can be fitted by least-squares curve fitting to calculate the satellite orbit state vector at any time during the imaging period. The third-order polynomial is used to fit the satellite trajectory (E.X. Chen, 2004). The expression is as follows equation (3) and (4), and the velocity vector can be obtained by calculate the first derivative of the position vector.

$$
\begin{gathered}
{\left[\begin{array}{l}
X_{S} \\
Y_{S} \\
Z_{S}
\end{array}\right]=\left[\begin{array}{c}
\sum_{k=0}^{k=2} a_{k} t^{k} \\
\sum_{k=0}^{k=2} b_{k} t^{k} \\
\sum_{k=0}^{k=2} c_{k} t^{k}
\end{array}\right]} \\
{\left[\begin{array}{l}
X_{V} \\
Y_{V} \\
Z_{V}
\end{array}\right]=\left[\begin{array}{c}
\sum_{k=0}^{k=2} k a_{k} t^{k-1} \\
\sum_{k=0}^{k=2} k b_{k} t^{k-1} \\
\sum_{k=0}^{k=2} k c_{k} t^{k-1}
\end{array}\right]}
\end{gathered}
$$

2.1.3. The Establishment of RD Model:The RD model establishes the correspondence between the SAR image points and the object points on the earth based on SAR geometric imaging principle (M. Shimada, 2010; Roth. A., 1993). The rigorous mathematical relation consist of the equation (5), the Doppler frequency equation (6) and the earth ellipsoid equation (7). $R_{t}=\left(X_{t}, Y_{t}, Z_{t}\right)$ and $V_{t}=\left(V_{x}, V_{y}, V_{z}\right)$ respectively represent the position vector and velocity vector of the object points in the coordinate system established in section 2.2.1, and the velocity vector $V_{t}$ equals zero vector. c equals the speed of light $\tau$ is the time delay that the pulse from the beginning of emitting to the end of receiving. $f_{d}$ represents the Doppler shift corresponding to the coordinate $(i, j)$ of the image, which can 
be obtained from the metadata by calculating. Re and $\mathrm{Rp}$ are the equatorial radius and polar radius of the Earth ellipsoid, all of which are known values. $\lambda$ is the wavelength of the microwave, which is a given value, and $h$ is the average elevation of the imaging area. In the process of locating using RD model, $\mathrm{R}(\mathrm{i}, \mathrm{j})$ and, $f_{d}$ can be obtained by calculation, and the position vector $R_{t}$ can be calculated by the AGM algorithm by solving these three equations.

$$
\begin{gathered}
R(i, j)=\sqrt{R_{s}^{2}-R_{t}^{2}}=c \cdot \tau / 2 \\
f_{d}=-\frac{2}{\lambda}\left(\frac{\left(R_{s}-R_{t}\right) \cdot\left(V_{s}-V_{t}\right)}{\sqrt{R_{s}^{2}-R_{t}^{2}}}\right) \\
\frac{X^{2}+Y^{2}}{\left(R_{e}+h\right)^{2}}+\frac{Z^{2}}{R_{p}^{2}}=1
\end{gathered}
$$

\subsection{AGM used for RD Model}

As shown in Figure 3, in the coordinate system established based on Figure 2. $\mathrm{O}^{\prime} \mathrm{N}$ is a arc passing $\mathrm{O}^{\prime}$, with $\mathrm{O}^{\prime}$ as the coordinate origin. OS vector is regard as the $\mathrm{z}$ axis, and the direction of OS vector is positive direction. Assume that a vector i.e. $\mathrm{x}$ axis belongs to $\mathrm{OO}^{`} \mathrm{~N}$ plane and passes the point $\mathrm{O}^{`}$ and this vector is perpendicular to OS vector, and y-axis is the result of the right-handed rectangular coordinate system O'-xyz. The angle $\alpha$ represents the central angle $\angle \mathrm{TOO}^{\prime}$. Drawing a circle passing points $\mathrm{T}, \mathrm{O}$ and $\mathrm{O}^{\prime}$, and then the angle $\alpha$ is the arc TO' covers. The angle $\beta$ is the angle between the plane TOO 'and the plane OO'N. Suppose that an area under the stars is regarded as a spherical surface whose local radius is a constant i.e. $\mathrm{R}_{\mathrm{L}}$, so $\mathrm{OO}^{\prime}=\mathrm{OT}=\mathrm{R}_{\mathrm{L}}, \mathrm{R}_{\mathrm{L}}$ can be obtained according to the following equation (8). Within the triangle OST, angle $\alpha$ can be obtained with the help of equation (9).

$$
\begin{gathered}
R_{L}=\sqrt{\frac{R_{e}^{2} R_{p}^{2}}{R_{e}^{2} \sin ^{2} \delta_{s}+R_{p}^{2} \cos ^{2} \delta_{s}}} \\
\cos \alpha=\frac{R_{S}^{2}+R_{L}^{2}-R(i, j)^{2}}{2 R_{S} R_{L}}
\end{gathered}
$$

where $\delta_{\mathrm{s}}$ is the latitude of the center of the SAR image.

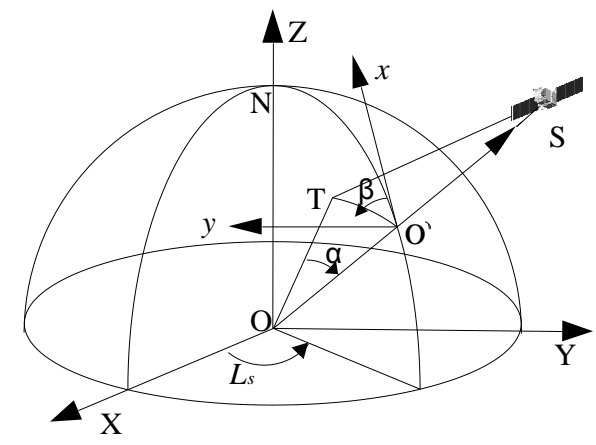

Figure 3. new local coordinate system $\mathrm{O}^{`}-\mathrm{xyz}$

AGM algorithm principle: the ground object point $\mathrm{T}$ is on the circle whose origin, radius and field angle are $\mathrm{O}, \mathrm{R}_{\mathrm{L}}$ and $\alpha$. If the value of $\beta$ is confirmed, then, the location of the T point will be fixed. If $\beta$ is changing and the position of $\mathrm{T}$ is also changing, then, the relative position of satellite and object point changes. Therefore, the Doppler frequency also changes. In other words, there exist a one-to-one mapping relationship between $\beta$ angle and $f_{d}$. As soon as $f_{d}$ is determined, $\beta$ is already fixed and $\mathrm{T}$ is fixed. $\beta$ can be calculated using the
Doppler frequency equation, then the position of $\mathrm{T}$ can also be calculated with the help of $\alpha$ and $\beta$. The following is the realization of the AGM algorithm.

(1) A new local coordinate system $\mathrm{O}^{-}$-xyz is created, then, transform the satellite's state vectors $R_{s}$ and $V_{s}$ which originally are in the coordinate system of $\mathrm{O}-\mathrm{XYZ}$ into $\mathrm{O}^{-}$-xyz coordinate system. The satellite's state vectors in $\mathrm{O}^{\prime}$-xyz coordinate system is denoted by $\mathrm{r}_{\mathrm{s}}$ and $\mathrm{v}_{\mathrm{s}}$, and $\mathrm{R}_{\mathrm{t}}$ is replaced with $r_{t}$ in the local coordinate system .

(2) Calculate the geocentric latitude and longitude $(1, b)$ of $\mathrm{O}$

$$
\begin{gathered}
\tan l=\frac{Y_{S}}{X_{S}} \\
\tan b=\frac{Z_{S}}{\sqrt{X_{S}^{2}+Y_{S}^{2}}}
\end{gathered}
$$

where $z_{s}=|R(i, j)|-R_{L}$

(3) Calculate $r_{\mathrm{s}}, \mathrm{v}_{\mathrm{s}}, r_{t}$

$$
\begin{gathered}
r_{s}=\left(0,0, z_{s}\right) \\
v_{s}=\left(x_{v}, y_{v}, z_{v}\right) \\
{\left[\begin{array}{l}
x_{v} \\
y_{v} \\
z_{v}
\end{array}\right]=\left[\begin{array}{c}
-X_{V} \sin (b) \cos (l)-Y_{V} \sin (b) \cos (l)+Z_{V} \cos (b) \\
X_{V} \sin (l)-Y_{V} \cos (l) \\
X_{V} \cos (b) \cos (l)+Y_{V} \cos (b) \sin (l)+Z_{V} \cos (b)
\end{array}\right]} \\
r_{t}=\left(x_{t}, y_{t}, z_{t}\right) \\
{\left[\begin{array}{l}
x_{t} \\
y_{t} \\
z_{t}
\end{array}\right]=\left[\begin{array}{c}
R_{L} \sin \alpha \cdot \cos \beta \\
R_{L} \sin \alpha \cdot \sin \beta \\
-R_{L}(1-\cos \alpha)
\end{array}\right]}
\end{gathered}
$$

(4) Calculate $\beta$ by Doppler equation

The Doppler frequency equation (17) is established in the $\mathrm{O}^{\prime}$-xyz coordinate system, and the above equations (12), (14) and (16) can be substituted into equation (17), then equation (18) is formed.

$$
f_{d}=-\frac{2}{\lambda}\left(\frac{\left(r_{s}-r_{t}\right) \cdot v_{s}}{\sqrt{r_{s}^{2}-r_{t}^{2}}}\right)
$$

$$
\begin{aligned}
& f_{d}=-\frac{2}{\lambda \sqrt{r_{s}^{2}-r_{t}^{2}}}\left(-x_{v}\left(R_{L} \sin \alpha \cdot \cos \beta\right)-y_{v}\left(R_{L} \sin \alpha \cdot \sin \beta\right)+\right. \\
& Z v(Z S+R L(1-\cos \alpha))
\end{aligned}
$$

Treat (18) as follows:

$$
\begin{aligned}
& K=\frac{2}{\lambda \sqrt{r_{s}^{2}-r_{t}^{2}}} \cdot\left(x_{v}\left(R_{L} \sin \alpha\right)\right) \\
& M=\frac{2}{\lambda \sqrt{r_{s}^{2}-r_{t}^{2}}} \cdot\left(y_{v}\left(R_{L} \sin \alpha\right)\right) \\
& N=-\frac{2}{\lambda \sqrt{r_{s}^{2}-r_{t}^{2}}} \cdot z_{v}\left(Z_{S}+R_{L}(1-\cos \alpha)\right)-f_{d}
\end{aligned}
$$

So (18) can be simplified to (19):

$$
K \cos \beta+M \sin \beta+N=0
$$

Dealing with equation (19):

$$
\cos \beta=\frac{-K N \pm M \sqrt{K^{2}+M^{2}-N^{2}}}{K^{2}+M^{2}}
$$


Deciding the plus sign ' + ' or subtraction sign ' - ' in the equation (20) depending on the SAR observation mode. when in way of left look, '-' will be chosen, and when in way of right look, ' + ' will be chosen.

(5) Calculate the geocentric latitude and longitude (Lt, $\delta \mathrm{t})$ of T using $\alpha, \beta$.

After $\alpha$ and $\beta$ are obtained, the position of T can be uniquely determined. Using the longitude and latitude $(1, b)$ and equation (21), (22), the latitude and longitude of T can be calculated.

$$
\begin{gathered}
\cos \left(\frac{\pi}{2}-\delta_{t}\right)=\cos \alpha b+\sin \alpha \cos b \cos \beta \\
\cos \left(l-L_{t}\right)=\frac{\cos \alpha-\sin b \sin \delta_{t}}{\cos b \cos \delta_{t}}
\end{gathered}
$$

\subsection{IAGM for SAR Image Geolocation}

According to the flowchart shown in Figure 1, can we implement the geolocation of SAR image. The imaging parameters are provided by the SAR metadata. Selecting the pixel $(\mathrm{m}, \mathrm{n})$ which corresponds to the GCPs on the earth, and the pixel coordinate $(\mathrm{m}, \mathrm{n})$ should be converted to the image plane coordinate $(i, j)$ as the input of the IAGM for SAR image geolocation. Using the AGM to calculate the geocentric latitude and longitude of the selected pixel, and interpolate the elevation of pixel's geolocation result which is marked with $\mathrm{H}_{\mathrm{t}}$ with the help of DEM, then transform the latitude and longitude coordinates into the $\mathrm{O}-\mathrm{XYZ}$ coordinate system. The difference between the coordinates of pixel's geolocation result after being transformed and the accurate coordinates of the GCPs is expressed as the linear regression with the argument of pixel's geolocation result under the coordinate of O-XYZ. Travelling all the existing GCPs, and the coefficients of the linear regression can be calculated by these GCPs with the method of Least Square Adjustment. Finally, by selecting the point of interest on the SAR image and adding the coordinate difference to the result of the geolocation by using AGM, then, high precision geolocation results will be obtained. The specific process is as follows A to E.

(1) $\mathrm{N}$ obvious objects are selected as the GCPs on the SAR image, and their image coordinates $\left(\mathrm{i}_{\mathrm{n}}, \mathrm{j}_{\mathrm{n}}\right)$ can be obtained from SAR image, besides, selecting N GCPs corresponding to the GCPs on the SAR image from the existing orthophoto. we can acquire their latitudes and longitudes coordinates, and then calculate the geocentric Cartesian coordinates $\left(\mathrm{X}_{\mathrm{t}}^{\prime}, \mathrm{Y}_{\mathrm{t}}^{\prime}, \mathrm{Z}_{\mathrm{t}}\right){ }_{\mathrm{n}}$ by the inverse operation of equation (23).

(2) Calculate the coordinate $\left(X_{t}, Y_{t}, Z_{t}\right)_{n}$ of the selected control points in the SAR image using the AGM, and the coordinate correction value $(\Delta \mathrm{X}, \Delta \mathrm{Y}, \Delta \mathrm{Z})$ can be calculated, i.e. $\Delta \mathrm{X}=\mathrm{X}_{\mathrm{t}}^{\prime}-\mathrm{X}_{\mathrm{t}}, \Delta \mathrm{Y}=\mathrm{Y}_{\mathrm{t}}-\mathrm{Y}_{\mathrm{t}}, \Delta \mathrm{Z}=\mathrm{Z}_{\mathrm{t}}^{\prime}-\mathrm{Z}_{\mathrm{t}}$.

(3) Establish a linear regression equation between coordinate correction value $(\Delta \mathrm{X}, \Delta \mathrm{Y}, \Delta \mathrm{Z})$ and the geolocation results coordinate $\left(X_{t}, Y_{t}, Z_{t}\right)$ i.e. (24).

$$
\left[\begin{array}{l}
\Delta X \\
\Delta Y \\
\Delta Z
\end{array}\right]=\left[\begin{array}{c}
d_{0}+d_{1} \times X_{t}+d_{2} \times Y_{t}+d_{3} \times Z_{t} \\
e_{0}+e_{1} \times X_{t}+e_{2} \times Y_{t}+e_{3} \times Z_{t} \\
f_{0}+f_{1} \times X_{t}+f_{2} \times Y_{t}+f_{3} \times Z_{t}
\end{array}\right]
$$

where $\Delta \mathrm{X}, \Delta \mathrm{Y}, \Delta \mathrm{Z}$ represent the difference in $\mathrm{X}$ axis, $\mathrm{Y}$ axis and $\mathrm{Z}$ axis. $\mathrm{X}_{\mathrm{t}}, \mathrm{Y}_{\mathrm{t}}, \mathrm{Z}_{\mathrm{t}}$ are the position vector by using AGM under the WGS84 coordinate system, $d_{0}, d_{1}, \cdots d_{3}, e_{0}$, $e_{1}, \cdots e_{3}, f_{0}, f_{1}, \cdots f_{3}$ are the coefficients.

(4) The coefficient of equation (24) is calculated by using N (N $\geqq 4$ ) GCPs with the method of Least Square Adjustment.

(5) Calculate any object point of interest. We can obtain the more satisfied coordinate of the object points you are interested in. The high precision geolocation of SAR images is realized by adding the coordinate correction value to the geolocation result of the AGM, and the expression is as following equation (25).

$$
\left[\begin{array}{c}
X_{t} \\
Y_{t} \\
Z_{t}^{\prime}
\end{array}\right]=\left[\begin{array}{l}
X_{t} \\
Y_{t} \\
Z_{t}
\end{array}\right]+\left[\begin{array}{c}
\Delta X \\
\Delta Y \\
\Delta Z
\end{array}\right]
$$

where $X_{t}^{\prime}, Y_{t}^{\prime}, Z_{t}^{\prime}$ are the position vector calculated by using IAGM under the WGS84 coordinate system.

\section{EXPERIMENT AND DISCUSSION}

\subsection{Experiment Introduction}

For the SAR image geolocation algorithm proposed in this paper, experiment takes Radarsat-2 SLC image of Canada. The experimental data of image area covers Lingchuan County, Guilin, Guangxi and surrounding areas, including mountains, lakes, residential areas, farmland, highways, bridges and so on. The image consists of $6153 \times 3332$ pixels, whose resolution in along and across track direction are 5.2 meter and 7.6 meter.

The specific information about the imaging parameters are given in Table 1. In addition, the 30m SRTM DEM will be used Figure 4 shows the SAR raw image. The image appears to be elongated because of the foreshortening in along track direction.

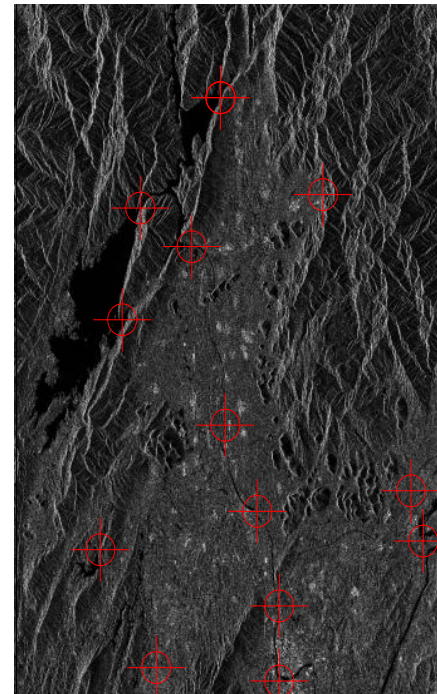

Figure 4. Radarsat-2 SLC image

\begin{tabular}{cc}
\hline Satellite sensor & Radarsat-2 \\
Date of acquisition (UTC) & June 21, 2015 \\
Acquisition mode & Stripmap \\
Image width $(\mathrm{km})$ & $25 \times 25$ \\
Image level & SLC \\
Descending/Ascending, & Ascending \\
Wavelength(meter) & 0.055466 \\
Angle of incidence & $34.62^{\circ}$ \\
Pulse Repetition Frequency(HZ) & 2770.771729 \\
Near slant range $(\mathrm{km})$ & 938.821821 \\
Right/Left look & Right \\
Center point coordinates & $25.526^{\circ} \mathrm{N} / 110.255^{\circ} \mathrm{E}$ \\
\hline
\end{tabular}

Table 1. imaging parameters information 


\subsection{Experimental Process and Result}

In order to evaluate the accuracy of the SAR image geolocation, 15 obvious ground points (accuracy within $3 \mathrm{~m}$ ) selected from the existing orthophoto are regarded as the check points. Besides, select 15 corresponding checking points on the SAR image. Experimental process: Firstly, respectively select 4, 5, $6 \cdots 12$ uniformly distributed GCPs displaied in Figure 4. Secondly, using the 9 sets of GCPs respectively to calculate the linear regression coefficients. Finally, calculate the geolocation results of the 15 checking points using AGM and IAGM.The final position vector for all geolocation is in the WGS84 geocentric Cartesian coordinate system. Due to the reference coordinate system of checking points choosed from the orthophoto is WGS84 coordinate Gaussian three-degree projection, to well evaluate the accuracy, the final position vector for geolocation will be converted to the reference coordinate system of checking points. Taking the position of checking points on the orthophoto as a standard, and comparing the position of the geolocation results with the standard position, and calculate the root mean square error(RMS). The RMS will be regarded as a reference for accuracy evaluation. The calculation formula of RMS are shown below Table 2. The specific process of using IAGM for the geolocation shows as follows:

(1) Determine the image plane coordinate $(i, j)$ of the pixel where the feature points needs to be deal with. In this paper the feature points refer to the GCPs.

(2) Calculate the geoocation parameters of each pixel according to the relevant parameters provided by the metadata.

(3) Use the AGM and IAGM algorithm to complete the 15 check points' geolocation according to steps (1) to (5) mentioned in section 2.3, moreover, the geolocation results of AGM and IAGM algorithm can be compared.

\subsection{Accuracy Evaluation of Experimental Results}

In order to evaluate the accuracy of experiments, Table 1 shows the geolocation results of the checking points selected on the orthophoto using AGM and IAGM algorithm. The results of the geolocation are under the WGS84 Gaussian three-degree projection rectangular coordinate system. The case of number of 0 GCPs corresponds to the results of using the AGM, and the other cases of 4 to 12 GCPs used for calculating linear regression coefficients to realize IAGM geolocation. Figure 5 shows the trend of geolocation accuracy respectively with 4 to 12 GCPs and linear regression .

By analyzing the data in Table 1, we can draw the following conclusions: (1) When there are not GCPs used for geolocation, i.e. using the AGM algorithm, the RMS in X and Y directions are $\pm 148.892 \mathrm{~m}$ and $\pm 189.344 \mathrm{~m}$. The plane RMS is $240.873 \mathrm{~m}$, and the geolocation accuracy is basically the same as the accuracy mentioned in paper. (2) When using four GCPs for geolocation, the errors in $\mathrm{X}$ and $\mathrm{Y}$ directions are obviously improved. The accuracy in the $\mathrm{X}$ direction is increased by $65.23 \%$, and accuracy in $\mathrm{Y}$ direction increased by $61.84 \%$. The plane geolocation accuracy increased by $63.10 \%$, and he reason is that the geolocation error caused by the satellite orbit error is

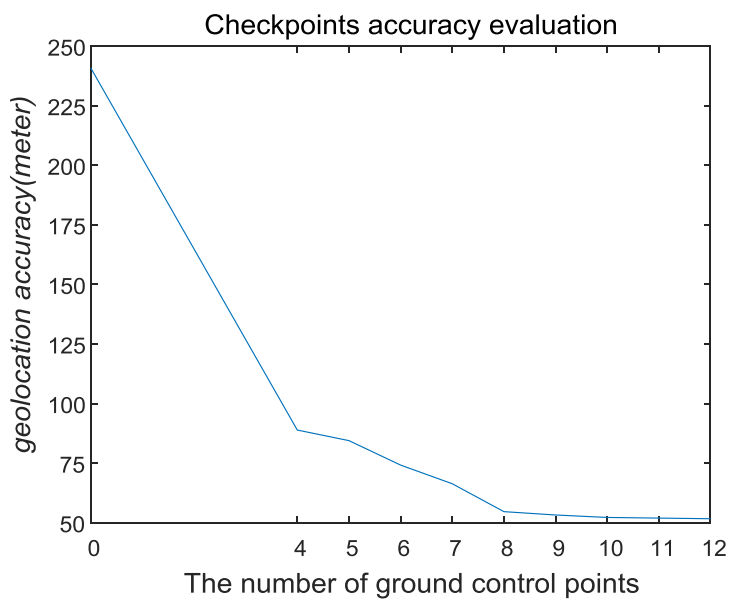

Figure 5. the trend of geolocation accuracy with control points

eliminated. (3) From table 2 and Figure 5 can be clearly seen, using four GCPs to improve the geolocation accuracy is the fastest. With the number of GCPs increaseing, the geolocation accuracy increases, but the increasing rate is not so big. When the number of GCPs reaches 12, the accuracy is unchanged, and the geolocation accuracy reaches $51.668 \mathrm{~m}$, which is why the author did not continue to increase the number of GCPs. Since the Earth's ellipsoid model can not be accurately expressed by an ideal mathematical model when locationg, and there is always existing a geolocation error

\begin{tabular}{|c|c|c|c|c|c|c|c|c|c|}
\hline \multirow{2}{*}{ GCPs } & \multicolumn{3}{|c|}{ Maximum residual(m) } & \multicolumn{3}{|c|}{ Minimum residual(m) } & \multicolumn{3}{|c|}{$\operatorname{RMS}(\mathrm{m})$} \\
\hline & $X$ & $\mathrm{Y}$ & plane & $\mathrm{X}$ & $\mathrm{Y}$ & plane & $\mathrm{X}$ & $\mathrm{Y}$ & Plane \\
\hline 0 & 214.526 & 324.217 & 388.765 & 36.597 & 78.323 & 86.451 & 148.892 & 189.344 & 240.873 \\
\hline 4 & 104.423 & 154.854 & 186.772 & 31.458 & 50.278 & 59.308 & 51.774 & 72.252 & 88.887 \\
\hline 5 & 107.254 & 152.037 & 186.061 & 29.645 & 50.314 & 58.398 & 48.325 & 69.247 & 84.442 \\
\hline 6 & 99.857 & 142.564 & 174.057 & 28.785 & 44.541 & 53.033 & 42.137 & 61.048 & 74.178 \\
\hline 7 & 92.658 & 154.498 & 180.153 & 27.887 & 47.356 & 54.957 & 37.275 & 54.889 & 66.349 \\
\hline 8 & 85.465 & 128.971 & 154.718 & 21.885 & 30.658 & 37.668 & 32.748 & 43.738 & 54.639 \\
\hline 9 & 84.574 & 121.564 & 148.090 & 21.063 & 35.612 & 41.374 & 31.558 & 42.867 & 53.231 \\
\hline 10 & 84.552 & 120.659 & 147.335 & 20.895 & 31.787 & 38.040 & 30.765 & 42.177 & 52.205 \\
\hline 11 & 84.401 & 120.467 & 147.091 & 20.221 & 30.784 & 36.831 & 30.689 & 41.893 & 51.931 \\
\hline 12 & 83.982 & 120.322 & 146.732 & 19.893 & 29.887 & 35.902 & 30.431 & 41.756 & 51.668 \\
\hline
\end{tabular}

Table 2. the accuracy of geolocation results respectively with 4 to12 GCPs and linear regression.

Where $R M S_{X}=\sqrt{\sum_{I=1}^{I=5}\left(X-X_{G}\right)^{2} / 5}, R M S_{Y}=\sqrt{\sum_{I=1}^{I=5}\left(Y-Y_{G}\right)^{2} / 5}, R M S_{\text {plane }}=\sqrt{R M S_{X}^{2}+R M S_{Y}^{2}}, \mathrm{X}, \mathrm{Y}$ are the position vector of check points calculated by using IAGM algorithm, $X_{G}, Y_{G}$ are the position vector obtained from orthophoto. 


\section{4 Discussion}

In view of the above experimental results, pixels of image geolocation errors can be roughly divided into three categories: (1) The limitations of AGM algorithm. A prerequisite for the establishment of the algorithm is to assume that the Earth's ellipsoid surface is spherical within a region of sub-satellite point. In fact it is not, then it implies the existence of geolocation error. (2) The defects of the RD model interior includes the errors of the earth ellipsoid model, the inaccuracy of the satellite orbit state vector, the errors of the slant range measurement and Doppler shift measurement error. (3) Limitation of linear regression and the errors of GCPs. In this paper, a rotating ellipsoid is used to approximate the shape of the earth. However, the real Earth is an irregular sphere, and the surface of the earth is rugged, undulating. It is difficult to describe it with a unified mathematical model. Therefore, the model used in this paper to participate in the geolocation calculation will inevitably result in errors in geolocation results. If the earth model can be effectively improved, the geolocation errors can be eliminated. The position of the satellite orbit directly affects the slant range equation and the Doppler frequency equation. When solving the geolocation model, even if the measurement of slant range and Doppler shift is accurate, however, since the satellite's position coordinate is inaccurate. It leads to the right side of the equation is inaccurate, either. Finally making the geolocation results inaccurate. Similarly, because of inaccuracies of the measurement of slant range and Doppler frequency, the value of the left side of the slant range equation and Doppler frequency equation is inaccurate. Even if the position coordinate of the satellite on the right side of the equation is accurate, the geolocation accuracy will still be affected.

Linear regression is a simulated process, not a rigorous mathematical correction model, and it can not exhaustively eliminate the geolocation error. Even though the number of GCPs are more than 12, improving IAGM geolocation results accuracy is very difficult.

\section{CONCLUSION}

In this paper, we aim to solve the RD model with the AGM algorithm. Although the computational efficiency is satisfied, the geolocation results are not so ideal. Due to the limitation of the algorithm and the internal structure of the RD model. Especially errors in the $\mathrm{Y}$ direction is always worse than the $\mathrm{X}$-direction. Therefore, a new method is proposed to optimize the geolocation results by using a linear regression and sparse GCPs. After the experiment of using IAGM with Radarsat-2 images, the result indicates that:

(1) If only using AGM to locate the target without using the GSPs, there will be a large geolocation error, especially there is a systematic offset in a certain direction.

(2) Using linear regression and sparse GCPs to correct the geolocation results of using the AGM can achieve better results, and geolocation accuracy can reach 50 meters.

(3) When the number of GCPs reaches 12, the geoocation accuracy will not improve with the increasing of GCPs.

The next step we can fix the number of GCPs, taking into account the Spatial distribution of the GCPs and the error of GCPs whether affect the accuracy of pixel geolocation results.

\section{ACKNOWLEDGEMENTS}

This paper is financially supported by the National Key Research and Development Program of China under Grant numbers 2016YFB0502500, the National Natural Science of China under Grant numbers 41431179, the State Oceanic Administration under Grant numbers 2014\#58, GuangXi Natural Science Foundation under grant numbers 2015GXNSFDA139032, Guangxi Science \& Technology Development Program under the Contract number GuiKeHe 14123001-4, and GuangXi Key Laboratory of Spatial Information and Geomatics Program under the Contract numbers 151400701, 151400712 and 163802512.

\section{REFERENCES}

A. Loew, W. Mauser, 2007. Generation of geometrically and radiometrically terrain corrected SAR image products, Remote Sensing of Environment, 106(2007), pp. 337-340.

E.X. Chen, 2004. Study on Ortho-rectification Methodology of Spaceborne Synthetic Radar Imagery[D], Chinese Academy of Forestry, pp. 31-35.

G. Zhou, W. Chen, and J. Kelmelis, 2005. A comprehensive study on urban aerial image orthorectification for national mapping program, IEEE Trans. on Geosciene and Remote Sensing, 43(9), pp. 2138-2147.

G. Zhou, T.Yue, Y. Shi, R. Zhang, J. Huang, 2016. Second Order Polynomial Equation-Based Block Adjustment for Orthorectification of DISP Imagery. Remote Sensing, 6(80); doi: $10.3390 / \mathrm{rs} 8080680$.

G. Zhang, X.W. Qin, 2013. Spaceborne SAR and InSAR data processing technology based on RPC model[M], Mapping Press, pp. 9-10.

G. Zhou, R. Zhang, and L. Jiang, 2017. On-board orthorectification of satellite image, Remote sensing doi, 9(9), $10.3390 /$ rs9090874.

H.H. Xu, 2005. Research on the target location Spaceborne Synthetic Radar Imagery[D], University of Chinese Academy of Science, pp. 45-46.

J.P. Zhou, 2000. Selection and optimization of the Location method for pixels in SAR imagery[J], Aerospace Shanghai, 2000(1), pp. 32-33.

J.P. Zhou, L.L. Tang, C.R. Li, 2001. Development of two practical RD location model and accuracy comparison between them[J], Journal of Remote Sensing, 5(3), pp. 191-192.

J.C. CURLANDER, 1982. Location of spaceborne SAR imagery[J], IEEE Transaction on Geoscience and Remote Sensing, 20(3), pp. 359.

K. G., Schuhr. W., 1988. Reliability of Radar image data, 16th ISPRS Congress, B9, Tokyo.

M. Shimada, 2010. Ortho-rectification and slope correc- tion of SAR data using DEM and Its accuracy evaluation, IEEE Journal Of Selected Topics In Applied Earth Observations And Remote Sensing, 3(4), pp. 657-659.

Roth. A., Craubner, A., \& Hüge 1, T., 1993. Standard geocoded ellipsoid corrected images, SAR Geocoding -Data and Systems, pp. 159-172.

X.K. Yuan, 1998. Target location method of Synthetic Aperture Radar satellite[J], Spacecraft Engineering, 7(2), pp. 23-26. 
Y.H. Zhang, 2001. Synthetic Aperture Radar Imaging Geometry Understanding and Processing[D], Wuhan University, pp. 35-36. 